\title{
Measurements of Natural Radioactivity of Industrial Raw Materials from the West of Saudi Arabia (Arabian Shield)
}

\author{
Safia H. Q. Hamidalddin \\ Physics Department, Girls Faculty of Science, King Abdulaziz University, Jeddah, Saudi Arabia \\ Email: Shamidaddin@kau.edu.sa, Safiahqh@yahoo.com \\ Received 1 October 2014; accepted 27 March 2015; published 30 March 2015 \\ Copyright (C) 2015 by author and Scientific Research Publishing Inc. \\ This work is licensed under the Creative Commons Attribution International License (CC BY). \\ http://creativecommons.org/licenses/by/4.0/

(c) ()

\section{Abstract}

Rock samples of industrial raw materials that are used in the building, from the West of Saudi Arabia (Arabian Shield), have been investigated using $\mathrm{X}$-ray diffraction to identify the mineral chemical composition. The concentrations of $\mathrm{Al} \%, \mathrm{Bi}, \mathrm{Pb}, \mathrm{Th}, \mathrm{U}$, and $\mathrm{K}$ in $\mathrm{ppm}$ were measured by atomic absorption analysis. The activity concentrations in $\mathrm{Bq} / \mathrm{kg}$ dry weight of ${ }^{226} \mathrm{Ra},{ }^{232} \mathrm{Th}$ and ${ }^{40} \mathrm{~K}$ were measured using gamma-ray spectrometry where the range and average were found to be (03.04 - 10.91) 07.90, (03.19 - 13.31) 09.14 and (95.59 - 361.52) 234.81 in Basalt, for Granite (33.81 - 89.13) 80.85, (28.42 - 112.77) 68.50 and (1260.13 - 1629.21) 1376.69, for Gold (00.78 11.84) 04.73, (01.48 - 4.69) 02.69 and (13.76 - 445.09) 197.58, for Andesite 05.72, 03.73 and 471.93, and for Marble 01.56, 01.38 and 10.15 respectively. Most results existing within the given values in building materials by UNSCEAR 1993 included $R a_{e q}(\mathrm{~Bq} / \mathrm{kg}), D(\mathrm{nGy} / \mathrm{h}), D_{\text {eff }}(\mathrm{mSv} / \mathrm{y}), H_{e x}$ and $H_{i n}$, which meant that it was safe for humans.

\section{Keywords}

Arabian, Shield Industrial Raw Materials, Harrat

\section{Introduction}

Natural radionuclides have always been present in all building materials such as rocks and soil which contain natural radionuclides, mainly of the ${ }^{226} \mathrm{Ra}$ and ${ }^{232} \mathrm{Th}$ series, and the radioactive isotope of potassium ${ }^{40} \mathrm{~K}$. Their specific activities vary depending on their origin and geochemical characteristics. The use of building materials rich with radionuclides may cause risks to human health. In Saudi Arabia, these building materials are mostly used for floors and external-internal cladding or in manufacture tiles, pipes and other corrosion resistant ceramic 
applications [1]. The study of radioactivity of building materials is important not only for the assessment of public dose rates and health risks but also for keeping reference-data record to document the possible changes in the environmental radioactivity and the nuclear activities [2]. Natural building stones are made from different types of material. Rocks of magmatic origin have lowest activities, sedimentary rocks such as marbles, limestone and various detrital contain small amounts of natural radionuclides and higher concentrations are generally found in acid magmatic rocks, especially in late-magmatic granites, and in some metamorphic rocks [3]. When uraniumradium $\left({ }^{238} \mathrm{U}-{ }^{226} \mathrm{Ra}\right.$ ) series decay, ${ }^{222} \mathrm{Ra}$ (radon) appears and its activity indoor is determined by building materials, water supply [8]. The presence of the radioisotopes in materials causes external and internal direct exposure to the people who live in the building. Studying radioactivity of industrial raw materials enables us to assess any possible radiological hazard to occupants of the dwelling by the use of such materials. ${ }^{226} \mathrm{Ra}$ and ${ }^{232} \mathrm{Th}$ are present in the Earth's crust in parts per million. Potassium is also present in the Earth's crust and $0.0118 \%$ of the total amount of potassium is ${ }^{40} \mathrm{~K}$. The measurement of radioactivity due to the presence of these nuclides in industrial raw materials is important for developing standards concerning the use of building materials [5].

This work presents the study of sixteen rock samples (industrial raw materials: Basalt, Granite, Gold, Andesite and Marble) from nine different sites using XR-D analyzer for the chemical and mineral composition, the analysis by atomic absorption for the $\mathrm{Al}, \mathrm{Bi}, \mathrm{Pb}, \mathrm{U}$, Th and $\mathrm{K}$ concentrations and for the radioactivity concentrations of ${ }^{226} \mathrm{Ra},{ }^{232} \mathrm{Th}$ and ${ }^{40} \mathrm{~K}$ by gamma-ray spectrometry. The radioactivity concentrations and their potential radiological hazards (radium equivalent activity $R a_{\text {eq }}$, the total absorbed dose rate $D$, the effective dose $D_{\text {eff }}$ and the external hazard index $H_{e x}$ and the internal hazard index $H_{i n}$ ) have been estimated and compared with the worldwide and the recommended limits from UNSCEAR data. These results are very important in the environmental radiological protection study, since such rocks are used as building and ornamental materials in Saudi Arabia.

\section{Analytical Technique}

\subsection{Study Area}

The Kingdom of Saudi Arabia occupies an area of more than two million square kilometers and its geological features allowed the formation of mineral deposits over the last billion years. The Arabian Shield is one of the Kingdom's geology. It is in the west running parallel to the Red Sea, covering one third of the Kingdom and is a major area for precious and basic minerals in addition to some industrial minerals [6]. Harrats cover an area over about 90,000 $\mathrm{km}^{2}$, and extending over parts of the Proterozoic Arabian shield and adjacent Phanerozoic rocks of the Arabian Platform and Red Sea basin. Most of industrial raw materials which used in building have been found in these harrats where Basalt samples were collected. The Arabian Shield has 4000 probable sites where Gold could be mined, but Mahd Al Thahab and Al Najadi are the most prominent (Gold samples were collected from these two mines). Al Quryyah in the province of Tabuk is characterized by the presence of high concentrations of uranium, thorium, rare elements ( $\mathrm{Ta}, \mathrm{Nb}, \mathrm{Zr}$ and $\mathrm{Y}$ ) and raw material (Granite were collected from this area). Andesite and Marble samples were collected from Bahrahand Madrekah (White Mountain) [7].

\subsection{Sample Collection and Measuring Methods}

Sixteen rock samples (7 Basalt, 4 Granite, 3 Gold, 1 Andesite, and 1 Marble) from nine different sites were collected at a randomly chosen point within the site area. Sampling points at each site were selected at a distance of $1-2 \mathrm{~km}$ from each other in order to cover a large area and to observe variation in radioactivity. The sites under investigation are shown in Figure 1 and their Sites, names, and locations are given in Table 1.

These samples were grounded, sieved by $1 \mathrm{~mm} \times 1 \mathrm{~mm}$, then dried for $24 \mathrm{~h}$ to remove moisture to $80^{\circ} \mathrm{C}$ not to lose the volatile ${ }^{137} \mathrm{Cs}$ or the natural polonium. Ten gm of the dried samples were analyzed by XRDmodelBurkerXR-DD8 Advance for the chemical and mineral composition; also ten gm of them were used for the analysis by atomic absorption model OPTIMA 4000 DV Series Perkin Elmer for the Al, Bi, Pb, U, Th, and K concentrations. For radiometric analysis, each dried sample was weighed and transferred to $640 \mathrm{cc}$ polyethylene Marinelli beakers then sealed and stored for 2 - 4 month to prevent the escape of Radon gas and to allow the attainment of radioactive secular equilibrium between ${ }^{238} \mathrm{U},{ }^{232} \mathrm{Th}$ and their progenies. The samples were analyzed non-destructively, using gamma-ray spectrometry with Canberra high purity germanium (HPGe) coaxial detector with relative efficiency of $25 \%$ and FWHM $2.0 \mathrm{keV}$ at $1332 \mathrm{keV}$, of ${ }^{60} \mathrm{Co}$. Genie 2000 basic spectroscopic software was installed in the computer for data acquisition and analysis. The system was calibrated for energy and absolute 


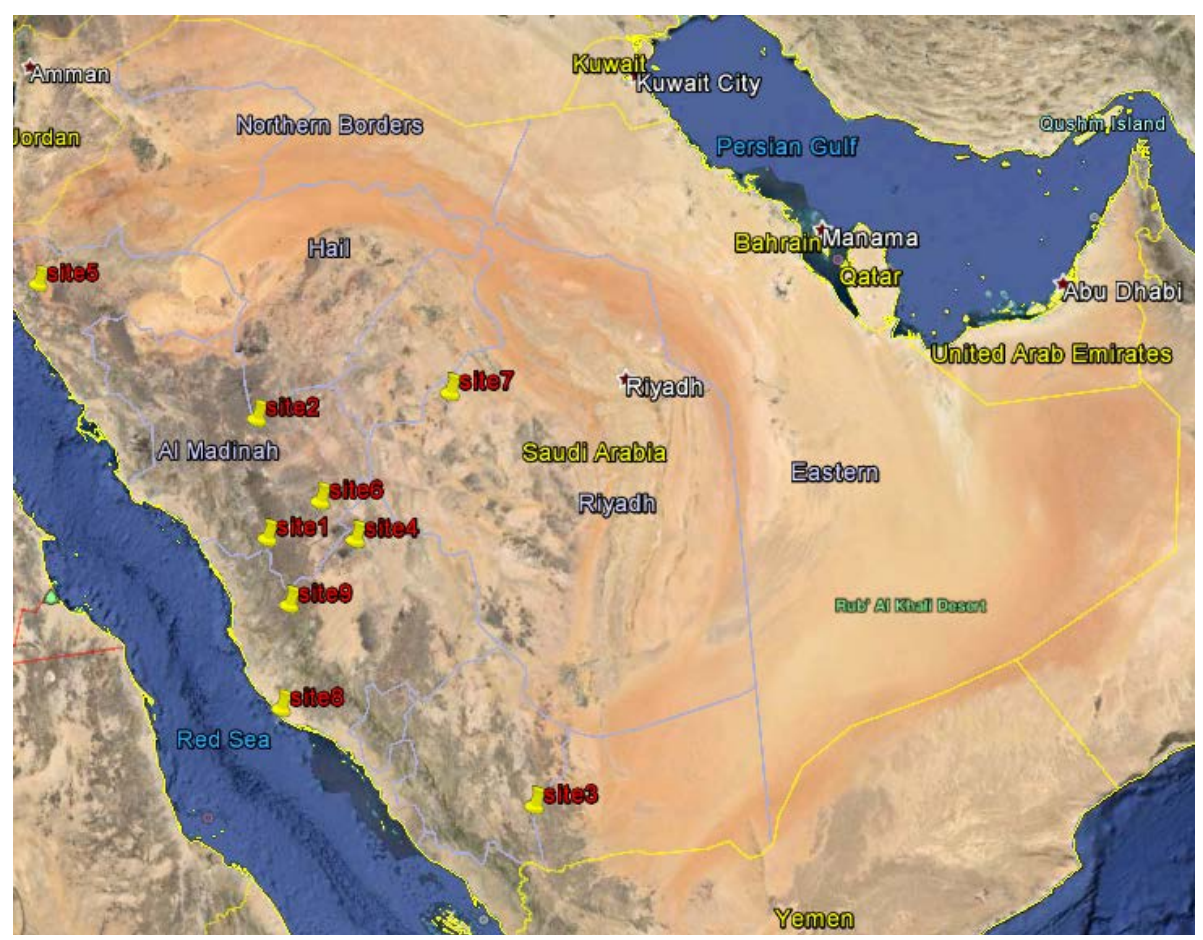

Figure 1. Represents samples sites.

Table 1. Sites name, and location of samples.

\begin{tabular}{|c|c|c|c|}
\hline Sampling site & Sample names & Location & Lat. and long. \\
\hline Site 1 & Basalt-1 + Basalt-2 & HarratRahat & $\begin{array}{l}\mathrm{N}: 23^{\circ} 05^{\prime} 00^{\prime \prime} \\
\mathrm{E}: 39^{\circ} 47^{\prime} 00^{\prime \prime}\end{array}$ \\
\hline Site 2 & Basalt-3 + Basalt-4 & HarratKhaybar & $\begin{array}{l}\mathrm{N}: 25^{\circ} 00^{\prime} 00^{\prime \prime} \\
\mathrm{E}: 39^{\circ} 55^{\prime} 01^{\prime \prime}\end{array}$ \\
\hline Site 3 & Basalt-5 & Harrat As Sirat & $\begin{array}{l}\mathrm{N}: 18^{\circ} 10^{\prime} 12^{\prime \prime} \\
\mathrm{E}: 43^{\circ} 34^{\prime} 12^{\prime \prime}\end{array}$ \\
\hline Site 4 & Basalt- $6+$ Basalt-7 & HarratKishb & $\begin{array}{l}\mathrm{N}: 22^{\circ} 48^{\prime} 0^{\prime \prime} \\
\mathrm{E}: 41^{\circ} 22^{\prime} 48^{\prime \prime}\end{array}$ \\
\hline Site 5 & $\begin{array}{l}\text { Granite-8 + Granite-9 + } \\
\text { Granite-10 + Granite-11 }\end{array}$ & Alquryyah & $\begin{array}{l}\mathrm{N}: 27^{\circ} 54^{\prime} 53^{\prime \prime} \\
\mathrm{E}: 35^{\circ} 47^{\prime} 52^{\prime \prime}\end{array}$ \\
\hline Site 6 & Gold-12 + Gold-13 & MahdAlThahab & $\begin{array}{l}\mathrm{N}: 23^{\circ} 30^{\prime} 12^{\prime \prime} \\
\mathrm{E}: 40^{\circ} 51^{\prime} 34^{\prime \prime}\end{array}$ \\
\hline Site 7 & Gold-14 & AlNajadi- & $\begin{array}{l}\mathrm{N}: 24^{\circ} 46^{\prime} 59^{\prime \prime} \\
\mathrm{E}: 43^{\circ} 29^{\prime} 57^{\prime \prime}\end{array}$ \\
\hline Site 8 & Andesite-15 & Bahrah & $\begin{array}{l}\mathrm{N}: 20^{\circ} 22^{\prime} 25^{\prime \prime} \\
\mathrm{E}: 39^{\circ} 30^{\prime} 52^{\prime \prime}\end{array}$ \\
\hline Site 9 & Marble-16 & Madrekah & $\begin{array}{l}\mathrm{N}: 21^{\circ} 58^{\prime} 45^{\prime \prime} \\
\mathrm{E}: 39^{\circ} 59^{\prime} 18^{\prime \prime}\end{array}$ \\
\hline
\end{tabular}

efficiency. The measurements were done for a time period of $82,800 \mathrm{sec}$. An empty polyethylene Marinelli beaker was placed in the detection system for this time period in order to collect the background count rates. Then, each sample was measured during a same accumulating time

\subsection{Calculations}

The concentration of ${ }^{226} \mathrm{Ra}$ was determined from the average concentrations of gamma-ray lines of energies 295.09 and $351.87 \mathrm{keV}$ of ${ }^{214} \mathrm{~Pb}$ and $609.31,1120.27$, and $1764.49 \mathrm{keV}$ of ${ }^{214} \mathrm{Bi}$ (since there is secular radioac- 
tivity equilibrium in ${ }^{226} \mathrm{Ra}$ series). ${ }^{232} \mathrm{Th}$, which it is in secular radioactivity equilibrium with its short half-life daughters, was determined from the average concentrations of ${ }^{228} \mathrm{Ac}$ (with gamma-ray lines at 338.32, 911.16, and $968.97 \mathrm{kev}$ ) and of ${ }^{212} \mathrm{Bi}$ at the line $727 \mathrm{keV}$, also ${ }^{208} \mathrm{Tl}$ with gamma-ray line $583.10 \mathrm{keV}$. The analysis of ${ }^{40} \mathrm{~K}$ concentrations was based on its single peak in the spectrum at energy $1460.80 \mathrm{kev}$, Determination of activity concentrations in $\mathrm{Bq} / \mathrm{kg}$ dry weight was calculated using Equation (1) [8].

$$
A=\frac{C}{m \beta \varepsilon}
$$

where: $C$ is the net peak area of specific gamma ray energy (count per second), $m$ is the mass of the samples in $(\mathrm{kg}), \quad \beta$ is the transition probability of gamma-decay, $\varepsilon$ is the detector absolute efficiency at the specific gamma-ray energy. Exposure to radiation has been defined in terms of the radium equivalent $R a_{e q} \mathrm{~Bq} / \mathrm{kg}$ which is calculated from Equation (2) [9].

$$
\mathrm{Ra}_{\mathrm{eq}}=C_{\mathrm{Ra}}+\left(C_{\mathrm{Th}} \times 1.43\right)+\left(C_{\mathrm{K}} \times 0.077\right)
$$

where: $C_{\mathrm{Ra}}, C_{\mathrm{Th}}$ and $C_{\mathrm{K}}$ are the concentrations in $\mathrm{Bq} / \mathrm{kg}$ dry weight for radium, thorium and potassium respectively. The total air absorbed dose rate $(\mathrm{nGy} / \mathrm{h})$ in the outdoor air at $1 \mathrm{~m}$ above the ground due to the activity concentrations of ${ }^{226} \mathrm{Ra},{ }^{232} \mathrm{Th}$ and ${ }^{40} \mathrm{~K}(\mathrm{~Bq} / \mathrm{kg})$ dry weight was calculated using Equation (3) [10] [11].

$$
D(\mathrm{nGy} / \mathrm{h})=0.462 C_{\mathrm{Ra}}+0.604 C_{\mathrm{Th}}+0.0417 C_{\mathrm{K}}
$$

where: $C_{\mathrm{Ra}}, C_{\mathrm{Th}}$, and $C_{\mathrm{K}}$ are the specific activities (concentrations) of ${ }^{226} \mathrm{Ra},{ }^{232} \mathrm{Th}$ and ${ }^{40} \mathrm{~K}$ in $\mathrm{Bq} / \mathrm{kg}$ dry weight respectively. The annual effective dose equivalent $D_{\text {eff }}(\mathrm{mSv} / \mathrm{y})$ in air was calculated using the values of the absorbed dose rate by applying the dose conversion factor of $0.7 \mathrm{~Sv} / \mathrm{Gy}$ and the outdoor occupancy factor of 0.2 (people spend about $20 \%$ of their life outdoor) the Annual Effective Dose (in $\mathrm{mSv} / \mathrm{y}$ ) received by population can be calculated using equation:

$$
D_{\text {eff }}(\mathrm{mSv} / \mathrm{y})=D(\mathrm{nGy} / \mathrm{h}) \times 8766 \mathrm{~h} \times 0.7(\mathrm{~Sv} / \mathrm{Gy}) \times 0.2 \times 10^{-6}
$$

where: $D(\mathrm{nG} / \mathrm{h})$ is the total air absorbed dose rate in the outdoor. $8,766 \mathrm{~h}$ is the number of hours in 1 year. $10^{-6}$ is conversion factor of nano and milli. To limit the annual external gamma-ray dose to $1.5 \mathrm{~Gy}$ for the samples under investigation, the external hazard index $\left(H_{e x}\right)$ is given by Equation (5) [12].

$$
H_{e x}=C_{\mathrm{Ra}} / 370+C_{\mathrm{Th}} / 259+C_{\mathrm{K}} / 4810
$$

The internal exposure to ${ }^{222} \mathrm{Rn}$ and its radioactive progeny is controlled by the internal hazard index $\left(H_{\text {in }}\right)$, which is given by Equation (6) [13].

$$
H_{i n}=C_{\mathrm{Ra}} / 185+C_{\mathrm{Th}} / 259+C_{\mathrm{K}} / 4810
$$

\section{Results and Discussion}

\subsection{XRD Analysis}

$\mathrm{X}$-ray diffraction is a non-destructive analytical technique, which provides detailed information about the atomic structure of crystalline substances, chemical composition, and physical properties of materials. It is a powerful tool in the identification of minerals in rocks and soils [14]. In the present study, the XRD results indicate that the main constituents (major) of these samples are Albite $\left(\mathrm{NaAlSi}_{3} \mathrm{O}_{8}\right)$, Anorthite $\left(\mathrm{CaAl}_{2} \mathrm{Si}_{2} \mathrm{O}_{8}\right)$, Augite $((\mathrm{Ca}, \mathrm{Na})$ $\left.\left.(\mathrm{Mg}, \mathrm{Fe}, \mathrm{Al}, \mathrm{Ti})(\mathrm{Si}, \mathrm{Al})_{2} \mathrm{O}_{6}\right)\right)$ in Basalt, Albite $\left(\mathrm{NaAlSi}_{3} \mathrm{O}_{8}\right)$, Microcline $\left(\mathrm{KAlSi}_{3} \mathrm{O}_{8}\right)$, Quartz $\left(\mathrm{SiO}_{2}\right)$ in Granite, Quartz $\left(\mathrm{SiO}_{2}\right)$ in Gold, Albite $\left(\mathrm{NaAlSi}_{3} \mathrm{O}_{8}\right)$, Clinochlore $\left(\mathrm{MgFe}^{2+}\right)_{5} \mathrm{Si}_{3} \mathrm{Al}_{2} \mathrm{O}_{10}(\mathrm{OH})_{8}$, Quartz $\left(\mathrm{SiO}_{2}\right)$ in Andesite, and Dolomite $\mathrm{CaMg}\left(\mathrm{CO}_{3}\right)_{2}$ in Marble. Small amount (Minor) and (Trace) minerals are presented as well. The mineral constituents of 16 samples analyzed by XRD spectrometer are shown in Table 2. Table 3 represents the mineral chemical composition and its description.

\subsection{Atomic Absorption Spectroscopy}

Table 4 gives concentrations of $\mathrm{Al} \%, \mathrm{Bi}, \mathrm{Pb}, \mathrm{Th}, \mathrm{U}$, and $\mathrm{K}$ in ppm by atomic absorption analysis. For Basalt 
Table 2. The mineral constituents of 16 samples analyzed by XRD spectrometer.

\begin{tabular}{|c|c|c|c|}
\hline Sam. no. & Major & Minor & Trace \\
\hline Basalt-1 & ANORTHITE & $\begin{array}{l}\text { CALCITE, MAGNETITE, } \\
\text { CLINOCHLORE, HALITE }\end{array}$ & $\begin{array}{l}\text { QUARTZ, AUGITE, } \\
\text { MONTMORILLONITE, OFFRETITE }\end{array}$ \\
\hline Basalt-2 & $\begin{array}{l}\text { ANORTHITE, } \\
\text { AUGITE }\end{array}$ & $\begin{array}{l}\text { MAGNETITE, CALCITE, } \\
\text { CLINOCHLORE }\end{array}$ & $\begin{array}{l}\text { HALITE, QUARTZ, BIOTITE, } \\
\text { PARGASITE, MONTMORILLONITE }\end{array}$ \\
\hline Basalt-3 & ANORTHITE & AUGITE, CALCITE & $\begin{array}{l}\text { HALITE, MAGNETITE, QUARTZ, } \\
\text { ZIRCON, NONTRONITE }\end{array}$ \\
\hline Basalt-4 & ANORTHITE & AUGITE, CALCITE & $\begin{array}{l}\text { HEMATITE, QUARTZ, ZIRCON, } \\
\text { BIOTITE, CLINOCHLORE, }\end{array}$ \\
\hline Basalt-5 & ANORTHITE & DIOPSIDE, MAGNETITE & $\begin{array}{l}\text { QUARTZ, HYDROGROSSULAR, } \\
\text { ZAKHAROVITE, CLINOCHLORE }\end{array}$ \\
\hline Basalt-6 & ANORTHITE & $\begin{array}{l}\text { DIOPSIDE, MAGNETITE, } \\
\text { CALCITE }\end{array}$ & $\begin{array}{l}\text { QUARTZ, BIOTITE, MONTMORILLONITE, } \\
\text { HYDROGROSSULAR }\end{array}$ \\
\hline Basalt-7 & ALBITE, ANORTHITE & $\begin{array}{l}\text { AUGITE, MAGNETITE, } \\
\text { CALCITE }\end{array}$ & BIOTITE, CLINOCHLORE, NONTRONITE \\
\hline Granite-8 & QUARTZ, ALBITE & MICROCLINE & $\begin{array}{l}\text { MAGNETITE, BIOTITE, CLINOCHLORE, } \\
\text { MONTMORILLONITE }\end{array}$ \\
\hline Granite-9 & $\begin{array}{l}\text { ALBITE, QUARTZ, } \\
\text { MICROCLINE, }\end{array}$ & CLINOCHLORE & $\begin{array}{l}\text { CALCITE, MAGNETITE, GOETHITE, } \\
\text { HEMATITE, ZIRCON }\end{array}$ \\
\hline Granite-10 & QUARTZ, ALBITE & MICROCLINE & $\begin{array}{l}\text { ZIRCO, CALCITE, MAGNETITE, } \\
\text { BIOTITE, CORDIERITE }\end{array}$ \\
\hline Granite-11 & QUARTZ, ALBITE & MICROCLINE, PARGASITE & $\begin{array}{l}\text { CALCITE, AUGITE, MAGNETITE, } \\
\text { ZIRCON, CLINOCHLORE, RUIZITE }\end{array}$ \\
\hline Gold-12 & QUARTZ & CLINOCHLORE & CALCITE, MAGNESIOHORNBLENDE \\
\hline Gold-13 & QUARTZ & CLINOCHLORE & $\begin{array}{l}\text { CALCITE, MICROCLINE, } \\
\text { MUSCOVITE, ZIRCON }\end{array}$ \\
\hline Gold-14 & QUARTZ & CALCITE, BIOTITE & $\begin{array}{l}\text { ALBITE, RUZITE, ANTIGORITE, } \\
\text { CHIAVENITE }\end{array}$ \\
\hline Andesite-15 & $\begin{array}{l}\text { QUARTZ, ALBITE, } \\
\text { CLINOCHLORE }\end{array}$ & BIOTITE, CALCITE & $\begin{array}{l}\text { MICROCLINE, ZIRCON, AUGITE, MAGNETITE, } \\
\text { MONTMORILLONITE, KARPINSKITE }\end{array}$ \\
\hline Marble-16 & DOLOMITE & CALCITE & ALBITE, QUARTZ, NONTRONITE, RIEBECKITE \\
\hline
\end{tabular}

samples, the concentrations of aluminum range from $8.17 \%$ to $9.42 \%$, the stable Lead $\left({ }^{208} \mathrm{~Pb},{ }^{206} \mathrm{~Pb}\right.$, and $\left.{ }^{207} \mathrm{~Pb}\right)$ vary from LDL $(<7.5)$ to $9.75 \mathrm{ppm}$, thorium vary from $\mathrm{LDL}(<1)$ to $3.75 \mathrm{ppm}$ (from $<4.07$ to $15.24 \mathrm{~Bq} / \mathrm{kg}$ ), potassium range from 3240 to $10440 \mathrm{ppm}$ (from 100.31 to 323.22). For Granite, the concentrations of Aluminum range from $6.23 \%$ to $6.95 \%$, lead vary from 20.23 to $40.53 \mathrm{ppm}$, thorium vary from 9.01 to 23.42 ppm (from 36.63 to $95.20 \mathrm{~Bq} / \mathrm{kg}$ ), potassium range from 31,960 to $40,600 \mathrm{ppm}$ (from 989.47 to $1256.66 \mathrm{~Bq} / \mathrm{kg}$ ). For Gold, the concentrations of aluminum range from $1.29 \%$ to $2.71 \%$, lead range from $<7.5$ to 42.86 ppm, thorium vary from $<1$ to $1.49 \mathrm{ppm}$ (from $<4.07$ to 6.06 ), potassium range from 1100 to $10,700 \mathrm{ppm}$ (from 34.06 to 331.27 $\mathrm{Bq} / \mathrm{kg}$ ) For Andesite, values of the concentrations are of aluminum 9.58, lead 11.08, thorium $<1 \mathrm{ppm}(<4.07$ $\mathrm{Bq} / \mathrm{kg})$ and $11,800 \mathrm{ppm}(365.33 \mathrm{~Bq} / \mathrm{kg})$. For Marble, the concentrations are of aluminum 0.04\%, Lead $<7.5 \mathrm{ppm}$, thorium $<1 \mathrm{ppm}(<4.07 \mathrm{~Bq} / \mathrm{kg})$ and potassium $140 \mathrm{ppm}(4.33 \mathrm{~Bq} / \mathrm{kg})$. Both bismuth $\left({ }^{209} \mathrm{Bi}\right)$ and uranium are LDL $(<10$ and $<5$ ppm respectively) for all samples except sample Gold-12 with value 13.76 ppm and sample Gold-13 with value $14.31 \mathrm{ppm}$. Conversion factors are $1 \mathrm{ppm}=4.06 \mathrm{~Bq} / \mathrm{kg}$ for ${ }^{232} \mathrm{Th}$ and $32.3 \mathrm{ppm}=1 \mathrm{~Bq} / \mathrm{kg}$ for ${ }^{40} \mathrm{~K}[17]$. Table 4 and Table 5 show that atomic absorption analysis results are in a good agreement with those obtained by gamma spectrometry measurements for thorium and for potassium. So, the atomic absorption analysis method is important for geochemical analysis to determine the concentrations of elements appearing in the examined minerals. 
Table 3. The mineral chemical composition and its description [15] [16].

\begin{tabular}{|c|c|c|c|}
\hline $\begin{array}{l}\text { Mineral/chemical } \\
\text { composition }\end{array}$ & Description & $\begin{array}{l}\text { Mineral/chemical } \\
\text { composition }\end{array}$ & Description \\
\hline Albite $\mathrm{NaAlSi}_{3} \mathrm{O}_{8}$ & $\begin{array}{l}\text { Sodium Plagioclase feldspar. } \\
\text { Magmatic and pegmatitic rocks. }\end{array}$ & $\begin{array}{l}\text { Magnesiohornblende } \\
\mathrm{Ca}_{2}\left[\mathrm{Mg}_{4}\left(\mathrm{Al}, \mathrm{Fe}^{3+}\right)\right] \mathrm{Si}_{7} \mathrm{AlO}_{22}(\mathrm{OH})_{2}\end{array}$ & $\begin{array}{l}\text { Igneous and } \\
\text { metamorphic rocks. }\end{array}$ \\
\hline Anorthite $\mathrm{CaAl}_{2} \mathrm{Si}_{2} \mathrm{O}_{8}$ & $\begin{array}{l}\text { Magmatic and } \\
\text { metamorphic rocks. }\end{array}$ & $\begin{array}{l}\text { Magnetite (lodestone) } \\
\mathrm{Fe}_{2}^{3+} \mathrm{Fe}^{2+} \mathrm{O}_{4}\end{array}$ & $\begin{array}{l}\text { mineral in igneous and } \\
\text { metamorphic rocks. }\end{array}$ \\
\hline Antigorite $\left(\mathrm{MgFe}^{2+}\right)_{3} \mathrm{Si}_{2} \mathrm{O}_{5}(\mathrm{OH})_{4}$ & $\begin{array}{l}\text { Common in regional and contact } \\
\text { metamorphosed serpentinites. }\end{array}$ & Microcline $\mathrm{KAlSi}_{3} \mathrm{O}_{8}$ & $\begin{array}{l}\text { Granitic pegmatites, } \\
\text { hydrothermal and } \\
\text { metamorphic rocks. }\end{array}$ \\
\hline $\begin{array}{l}\text { Augite-a pyroxene } \\
(\mathrm{Ca}, \mathrm{Na})(\mathrm{Mg}, \mathrm{Fe}, \mathrm{Al}, \mathrm{Ti})(\mathrm{Si}, \mathrm{Al})_{2} \mathrm{O}_{6}\end{array}$ & $\begin{array}{l}\text { Ferromagnesian silicate. Basic } \\
\text { igneous and metamorphic rocks. }\end{array}$ & $\begin{array}{l}\text { Montmorillonite } \\
\mathrm{NaCaAl}_{2} \mathrm{Si}_{4} \mathrm{O}_{10}(\mathrm{OH})_{2}\left(\mathrm{H}_{2} \mathrm{O}\right)_{10}\end{array}$ & $\begin{array}{l}\text { Absorbing water } \\
\text { and expanding. }\end{array}$ \\
\hline $\begin{array}{l}\text { Biotite-Black mica } \\
\mathrm{K}\left(\mathrm{MgFe}^{2+}\right)_{3} \mathrm{AlSi}_{3} \mathrm{O}_{10}(\mathrm{OH}, \mathrm{F})_{2}\end{array}$ & $\begin{array}{l}\text { Granitic rocks. Forms a } \\
\text { series with phlogopite. }\end{array}$ & $\begin{array}{l}\text { Muscovite } \\
\mathrm{KAl}_{2}\left(\mathrm{Si}_{3} \mathrm{Al}\right) \mathrm{O}_{10}(\mathrm{OH}, \mathrm{F})_{2}\end{array}$ & Granites and pegmatites. \\
\hline Calcite $\mathrm{CaCo}_{3}$ & Found in all kind of rocks. & $\begin{array}{l}\text { Nontronite } \\
\mathrm{NaFe}_{2}^{3+} \mathrm{Si}_{3} \mathrm{AlO}_{10}(\mathrm{OH})_{2} 4\left(\mathrm{H}_{2} \mathrm{O}\right)\end{array}$ & $\begin{array}{l}\text { It is the iron (III) rich member } \\
\text { of the smectite group of clay } \\
\text { minerals. }\end{array}$ \\
\hline $\begin{array}{l}\text { Chiavennite } \\
\mathrm{CaMnBe}_{2} \mathrm{Si}_{5} \mathrm{O}_{13}(\mathrm{OH})_{2} \cdot 2\left(\mathrm{H}_{2} \mathrm{O}\right)\end{array}$ & $\begin{array}{l}\text { Vitreous, massive, orange inter } \\
\text { grown in white quartz matrix. }\end{array}$ & $\begin{array}{l}\text { Offretite } \\
\left(\mathrm{K}_{2}, \mathrm{Ca}, \mathrm{Mg}\right)_{2} \cdot 5 \mathrm{Al}_{5} \mathrm{Si}_{13} \mathrm{O}_{36} \cdot 15\left(\mathrm{H}_{2} \mathrm{O}\right)\end{array}$ & $\begin{array}{l}\text { Alteration of basalt with the } \\
\text { addition of potash. }\end{array}$ \\
\hline $\begin{array}{l}\text { Clinochlore } \\
\left(\mathrm{MgFe}^{2+}\right)_{5} \mathrm{Si}_{3} \mathrm{Al}_{2} \mathrm{O}_{10}(\mathrm{OH})_{8}\end{array}$ & $\begin{array}{l}\text { Alteration mineral. } \\
\text { Metamorphic rock. }\end{array}$ & $\begin{array}{l}\text { Pargasite } \\
\mathrm{NaCa}_{2} \mathrm{Mg}_{3} \mathrm{Fe}^{2+} \mathrm{Si}_{6} \mathrm{Al}_{3} \mathrm{O}_{22}(\mathrm{OH})_{2}\end{array}$ & $\begin{array}{l}\text { A complex inosilicate mineral } \\
\text { of the amphibole group. }\end{array}$ \\
\hline Diopside $\mathrm{CaMg}\left(\mathrm{Si}_{2} \mathrm{O}_{6}\right)$ & $\begin{array}{l}\text { Basic and ultrabasic igneous } \\
\text { and metamorphic rocks. }\end{array}$ & Quartz $\left(\mathrm{SiO}_{2}\right)$ & $\begin{array}{l}\text { It is a component of almost } \\
\text { every rock type. }\end{array}$ \\
\hline Dolomite $\mathrm{CaMg}\left(\mathrm{CO}_{3}\right)_{2}$ & $\begin{array}{l}\text { Anhydrous carbonate mineral } \\
\text { (sedimentary carbonate rock). }\end{array}$ & $\begin{array}{l}\text { Ruizite } \\
\mathrm{CaMn}^{3+} \mathrm{Si}_{2} \mathrm{O}_{6}(\mathrm{OH}) \cdot 2\left(\mathrm{H}_{2} \mathrm{O}\right)\end{array}$ & $\begin{array}{l}\text { Cooling of a high temperature } \\
\text { calc-silicate rock oxidizing } \\
\text { onditions. }\end{array}$ \\
\hline Goethite $\mathrm{Fe}^{3+} \mathrm{O}(\mathrm{OH})$ & $\begin{array}{l}\text { Common in iron ore deposits. } \\
\text { Concentrated in laterite soils. }\end{array}$ & $\begin{array}{l}\text { Riebeckite } \\
\mathrm{Na}_{2} \mathrm{Fe}_{3}^{2+} \mathrm{Fe}_{2}^{3+}\left(\mathrm{Si}_{8} \mathrm{O}_{22}\right)(\mathrm{OH})_{2}\end{array}$ & $\begin{array}{l}\text { A sodium-rich member of the } \\
\text { amphibole group of silicate } \\
\text { minerals. }\end{array}$ \\
\hline Halite $\mathrm{NaCl}$ & $\begin{array}{l}\text { Marine or continental } \\
\text { Evaporite deposits. }\end{array}$ & $\begin{array}{l}\text { KARPINSKITE } \\
\mathrm{NiMgSi}_{2} \mathrm{O}_{5}(\mathrm{OH})_{2}\end{array}$ & $\begin{array}{l}\text { An inadequately described } \\
\text { mineral. }\end{array}$ \\
\hline Hematite $\mathrm{Fe}_{2} \mathrm{O}_{3}$ & $\begin{array}{l}\text { Magmatic, sedimentary } \\
\text { memetamorphic. }\end{array}$ & $\begin{array}{l}\text { Zakharovite } \\
\mathrm{Na}_{4} \mathrm{Mn}_{5}^{2+} \mathrm{Si}_{10} \mathrm{O}_{24}(\mathrm{OH})_{6} \cdot 6\left(\mathrm{H}_{2} \mathrm{O}\right)\end{array}$ & Differentiated alkalic massifs. \\
\hline $\begin{array}{l}\text { Hydrogrossular } \\
\mathrm{Ca}_{3} \mathrm{Al}_{2}\left(\mathrm{SiO}_{4}\right)_{3}(\mathrm{OH})_{4}\end{array}$ & $\begin{array}{l}\text { Found in massive crystal } \\
\text { grown in with idocrase. }\end{array}$ & Zircon $\mathrm{ZrSiO}_{4}$ & $\begin{array}{l}\text { Zircon is a mineral } \\
\text { (group of nesosilicates). }\end{array}$ \\
\hline
\end{tabular}

Table 4. Concentrations of Al, Bi, Pb, U, Th and K measured by Atomic Absorption.

\begin{tabular}{|c|c|c|c|c|c|c|c|c|}
\hline \multirow{2}{*}{$\begin{array}{c}\text { Elements } \\
\text { Units }\end{array}$} & \multirow{2}{*}{$\begin{array}{l}\text { Al } \\
\%\end{array}$} & \multirow{2}{*}{$\begin{array}{c}\text { Bi } \\
\text { ppm }\end{array}$} & \multirow{2}{*}{$\begin{array}{c}\text { Pb } \\
\text { ppm }\end{array}$} & \multirow{2}{*}{$\begin{array}{c}\mathrm{U} \\
\text { ppm }\end{array}$} & \multicolumn{2}{|c|}{ Th } & \multicolumn{2}{|c|}{$\mathbf{K}$} \\
\hline & & & & & ppm & $\mathrm{Bq} / \mathbf{k g}$ & ppm & $\mathbf{B q} / \mathbf{k g}$ \\
\hline DL. & 0.01 & 10 & 7.5 & 5 & 1 & 4.07 & 10 & 0.31 \\
\hline Basalt-1 & 8.94 & $<10$ & 8.91 & $<5$ & 2.87 & 11.67 & 10,440 & 323.22 \\
\hline Basalt-2 & 8.17 & $<10$ & 9.75 & $<5$ & 3.75 & 15.24 & 6540 & 202.48 \\
\hline Basalt-3 & 8.75 & $<10$ & $<7.5$ & $<5$ & $<1$ & $<4.07$ & 3240 & 100.31 \\
\hline Basalt-4 & 8.18 & $<10$ & 8.92 & $<5$ & 2.12 & 08.62 & 9600 & 297.21 \\
\hline Basalt-5 & 9.42 & $<10$ & 7.75 & $<5$ & $<1$ & $<4.07$ & 4000 & 123.84 \\
\hline Basalt-6 & 8.95 & $<10$ & 8.83 & $<5$ & $<1$ & $<4.07$ & 4260 & 131.89 \\
\hline Basalt-7 & 8.37 & $<10$ & 8.38 & $<5$ & 1.52 & 06.18 & 6660 & 206.19 \\
\hline Granie-8 & 6.95 & $<10$ & 40.53 & $<5$ & 20.00 & 81.30 & 31,960 & 989.47 \\
\hline Granie-9 & 6.84 & $<10$ & 21.06 & $<5$ & 23.42 & 95.20 & 39,040 & 1208.67 \\
\hline Granie-10 & 6.50 & $<10$ & 23.93 & $<5$ & 9.01 & 36.63 & 32,600 & 1009.29 \\
\hline Granie-11 & 6.23 & $<10$ & 20.23 & $<5$ & 18.94 & 76.99 & 40,600 & 1256.66 \\
\hline Gold-12 & 1.29 & 13.76 & 42.86 & $<5$ & $<1$ & $<4.07$ & 1100 & 34.06 \\
\hline Gold-13 & 1.77 & 14.31 & 18.12 & $<5$ & 1.49 & 06.06 & 3720 & 115.17 \\
\hline Gold-14 & 2.71 & $<10$ & $<7.5$ & $<5$ & $<1$ & $<4.07$ & 10,700 & 331.27 \\
\hline Andesite-15 & 9.58 & $<10$ & 11.08 & $<5$ & $<1$ & $<4.07$ & 11,800 & 365.33 \\
\hline Marble-16 & 0.04 & $<10$ & $<7.5$ & $<5$ & $<1$ & $<4.07$ & 140 & 04.33 \\
\hline
\end{tabular}


Table 5. Activity concentrations in $(\mathrm{Bq} / \mathrm{kg})$ dry weight of ${ }^{226} \mathrm{Ra},{ }^{232} \mathrm{Th}$ and ${ }^{40} \mathrm{~K}$ in rock samples and the ${ }^{226} \mathrm{Ra} /{ }^{232} \mathrm{Th}$ activity ratio.

\begin{tabular}{|c|c|c|c|c|}
\hline \multirow{2}{*}{ Sample name } & \multicolumn{3}{|c|}{ Activity concentration $(\mathbf{B q} / \mathbf{k g}))$} & \multirow{2}{*}{$\begin{array}{c}\text { Activity ratios } \\
{ }^{226} \mathrm{Ra} /{ }^{232} \mathrm{Th}\end{array}$} \\
\hline & ${ }^{226} \mathrm{Ra}$ & ${ }^{232} \mathrm{Th}$ & ${ }^{40} \mathrm{~K}$ & \\
\hline Basalt-1 & $08.52 \pm 0.01$ & $11.15 \pm 0.02$ & $353.75 \pm 0.02$ & 0.76 \\
\hline Basalt-2 & $10.31 \pm 0.02$ & $10.68 \pm 0.03$ & $213.88 \pm 0.02$ & 0.97 \\
\hline Basalt-3 & $03.04 \pm 0.02$ & $03.19 \pm 0.02$ & $95.59 \pm 0.01$ & 0.95 \\
\hline Basalt-4 & $10.55 \pm 0.01$ & $13.31 \pm 0.03$ & $361.52 \pm 0.02$ & 0.79 \\
\hline Basalt-5 & $04.69 \pm 0.01$ & $07.57 \pm 0.02$ & $155.39 \pm 0.02$ & 0.62 \\
\hline Basalt-6 & $05.55 \pm 0.01$ & $06.40 \pm 0.02$ & $146.67 \pm 0.02$ & 0.87 \\
\hline Basalt-7 & $07.19 \pm 0.02$ & $08.51 \pm 0.03$ & $221.25 \pm 0.01$ & 0.85 \\
\hline Range & $03.04-10.91$ & $03.19-13.31$ & $95.59-361.52$ & $0.62-0.97$ \\
\hline Average & $\mathbf{0 7 . 9 0} \pm \mathbf{0 . 0 2}$ & $09.14 \pm 0.03$ & $234.81 \pm 0.02$ & 0.83 \\
\hline Granite-8 & $112.77 \pm 0.01$ & $82.72 \pm 0.03$ & $1263.40 \pm 0.01$ & 1.36 \\
\hline Granite-9 & $96.07 \pm 0.01$ & $89.13 \pm 0.02$ & $1629.21 \pm 0.01$ & 1.08 \\
\hline Granite-10 & $28.42 \pm 0.01$ & $33.81 \pm 0.03$ & $1260.13 \pm 0.02$ & 0.84 \\
\hline Granite-11 & $86.15 \pm 0.02$ & $68.34 \pm 0.02$ & $1354.10 \pm 0.01$ & 1.26 \\
\hline Range & $28.42-112.77$ & $33.81-89.13$ & $1260.13-1629.21$ & $0.84-1.36$ \\
\hline Average & $80.85 \pm \mathbf{0 . 0 1}$ & $68.50 \pm 0.03$ & $1376.69 \pm 0.1$ & 1.14 \\
\hline Gold-12 & $00.78 \pm 0.01$ & $01.48 \pm 0.02$ & $13.76 \pm 0.01$ & 0.53 \\
\hline Gold-13 & $01.58 \pm 0.01$ & $01.90 \pm 0.02$ & $133.89 \pm 0.01$ & 0.83 \\
\hline Gold-14 & $11.84 \pm 0.02$ & $04.69 \pm 0.02$ & $445.09 \pm 0.01$ & 2.53 \\
\hline Range & $00.78-11.84$ & $01.48-4.69$ & $13.76-445.09$ & $0.53-2.53$ \\
\hline Average & $\mathbf{0 4 . 7 3} \pm \mathbf{0 . 0 1}$ & $02.69 \pm 0.02$ & $197.58 \pm 0.01$ & 1.30 \\
\hline Andesite-15 & $05.72 \pm 0.01$ & $03.73 \pm 0.02$ & $471.93 \pm 0.01$ & 1.53 \\
\hline Marble-16 & $01.56 \pm 0.02$ & $01.38 \pm 0.02$ & $10.15 \pm 0.01$ & 1.13 \\
\hline
\end{tabular}

\subsection{Gamma Spectroscopy}

The measured concentrations in $(\mathrm{Bq} / \mathrm{kg})$ dry weight of natural radionuclides ${ }^{226} \mathrm{Ra},{ }^{232} \mathrm{Th}$ and ${ }^{40} \mathrm{~K}$ in sixteen studied samples are listed in Table 5 and shown in Figure 2. The concentrations in $\mathrm{Bq} / \mathrm{kg}$ dry weight of ${ }^{226} \mathrm{Ra}$ range from 03.04 to 10.91 with an average value of $07.90,{ }^{232} \mathrm{Th}$ concentrations in $\mathrm{Bq} / \mathrm{kg}$ dry weight range from 03.19 to 13.31 with an average value of 09.14 , whereas ${ }^{40} \mathrm{~K}$ concentrations in $\mathrm{Bq} / \mathrm{kg}$ dry weight exist in the range from 95.59 to 361.52 with an average value of 234.81 in Basalt samples. For Granite samples, the concentrations in $\mathrm{Bq} / \mathrm{kg}$ dry weight of ${ }^{226} \mathrm{Ra},{ }^{232} \mathrm{Th}$ and ${ }^{40} \mathrm{~K}$ range from 28.42 to 112.77 , from 33.81 to 89.13 , from 1260.13 to 1629.21 respectively and the average activity of ${ }^{226} \mathrm{Ra},{ }^{232} \mathrm{Th}$ and ${ }^{40} \mathrm{~K}$ are $80.85,68.50$ and 1376.69 respectively. The concentrations in $\mathrm{Bq} / \mathrm{kg}$ dry weight of ${ }^{226} \mathrm{Ra},{ }^{232} \mathrm{Th}$ and ${ }^{40} \mathrm{~K}$ in gold samples range from 00.78 to 11.84 , from 01.48 to 4.69 , and from 13.76 to 445.09 respectively. The activity concentrations in $\mathrm{Bq} / \mathrm{kg}$ dry weight of ${ }^{226} \mathrm{Ra}$, ${ }^{232} \mathrm{Th}$ as well as ${ }^{40} \mathrm{~K}$ for Andesite are 05.72, 03.73, 471.93 and for Marble are 01.56, 01.38, 10.15 respectively. The results showed that, the Granite samples have the highest radiation comparison with other samples, while the Marble sample has the lowest. Also the results showed that, Harrat As Sirat sample (Basalt-3) has the lowest activity concentrations of other Harrat and Al Najadi Gold mine sample (Gold-14) has the highest activity concentrations of MahdAlThahab mine samples (Gold 12 and 13). The results of the activity of ${ }^{226} \mathrm{Ra} /{ }^{232} \mathrm{Th}$ ratios indicate that in Basalt samples, with two exceptions 2 and $3,{ }^{232} \mathrm{Th}$ is in excess to ${ }^{226} \mathrm{Ra}$. This is due to weather- 


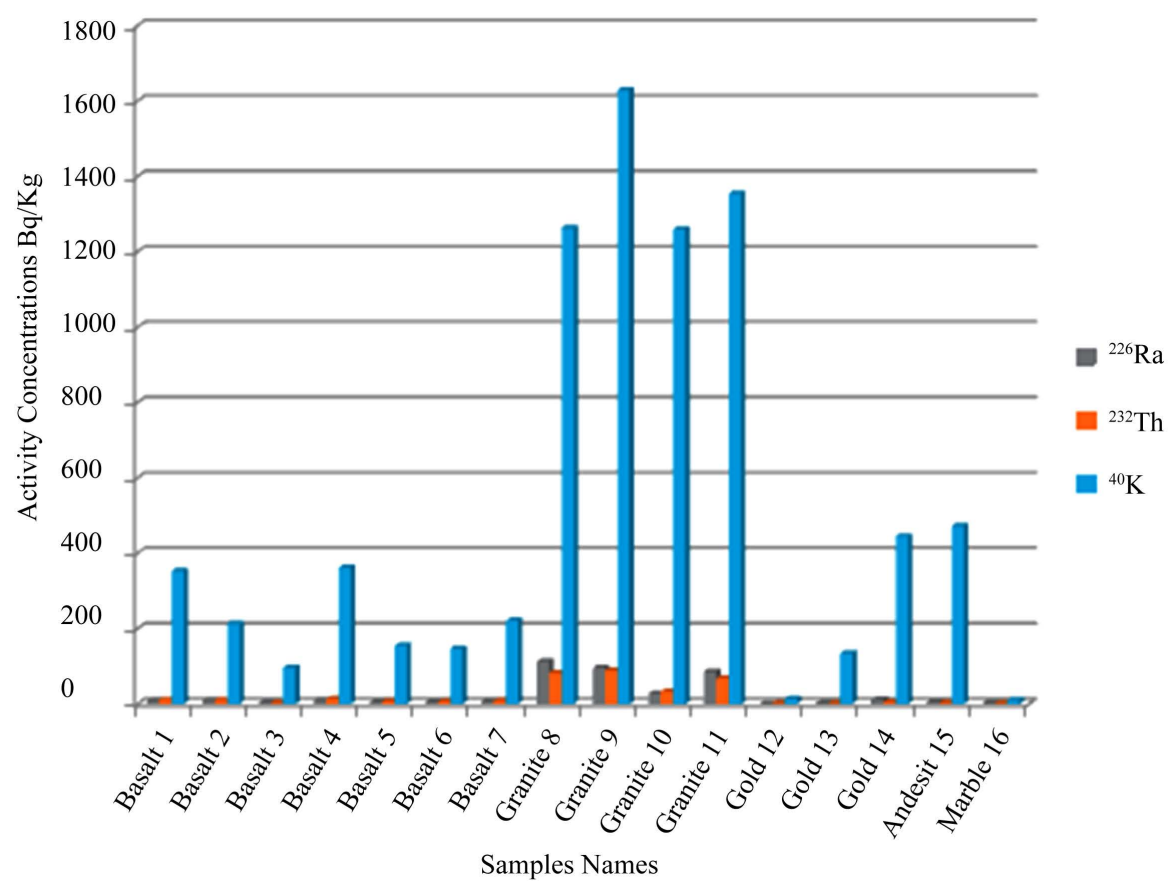

Figure 2. Activity concentrations in $(\mathrm{Bq} / \mathrm{kg})$ of ${ }^{226} \mathrm{Ra},{ }^{232} \mathrm{Th}$ and ${ }^{40} \mathrm{~K}$.

ing processes and water interaction with samples. For Granite samples, ${ }^{226} \mathrm{Ra}$ is in excess to ${ }^{232} \mathrm{Th}$ except sample 10. Gold has low activity of ${ }^{226} \mathrm{Ra} /{ }^{232} \mathrm{Th}$ ratios in two samples 12 and 13. Sample 14 (from AlNajadi) is enriched in ${ }^{226} \mathrm{Ra}\left({ }^{238} \mathrm{U}\right)$. For Andesite and Marble, activities of ${ }^{226} \mathrm{Ra} /{ }^{232} \mathrm{Th}$ ratios are high for ${ }^{226} \mathrm{Ra}$. In general, all results existed (except granite samples 8, 9 and 11, for ${ }^{226} \mathrm{Ra}$ and ${ }^{232} \mathrm{Th}$ and all Granite samples for ${ }^{40} \mathrm{~K}$ ) within the given values in building materials by UNSCEAR 1993: (50 Bq/kg for $\left.{ }^{226} \mathrm{Ra}\right),\left(50 \mathrm{~Bq} / \mathrm{kg}\right.$ for $\left.{ }^{232} \mathrm{Th}\right)$ and $(500 \mathrm{~Bq} / \mathrm{kg}$ for $\left.{ }^{40} \mathrm{~K}\right)[18]$.

\subsection{Radiation Hazard Indices}

It is important to evaluate the gamma radiation hazards by calculating the different radiation hazard indices. The radium equivalent concept allows describing the gamma radiation from radium, thorium and ${ }^{40} \mathrm{~K}$ in samples. Table 6 presents $R a_{e q}(\mathrm{~Bq} / \mathrm{kg}), D(\mathrm{nGy} / \mathrm{h}), D_{\text {eff }}(\mathrm{mSv} / \mathrm{y}), H_{e x}$ and $H_{i n}$ for studied samples. The radium equivalent activities $R a_{e q}(\mathrm{~Bq} / \mathrm{kg})$ of samples under investigation ranged from 14.96 to 57.42 with an average value of 36.58 for Basalt, 173.80 to 348.98 with an average value of 284.81 for Granite, 3.96 to 52.82 with an average value of 23.79 for Gold, 47.39 for Andesite and 4.32 for Marble. These calculated values of radium equivalent activities for all samples were below the recommended maximum value of $370 \mathrm{~Bq} / \mathrm{kg}$ where the highest value is 348.98 $\mathrm{Bq} / \mathrm{kg}$ from Alquryyah (Granite 9). For Basalt, the absorbed gamma dose rates $D(\mathrm{nGy} / \mathrm{h}$ ) ranged from 07.25 to 27.74 with an average value of 17.61 and for Granite, it ranged between 85.22 and 165.02 with an average vale 135.17. These calculated values were higher than the estimate of average global terrestrial radiation of 55 (nGy/h) UNSCEER. Gold samples had values of the absorbed gamma dose rates $D(\mathrm{nGy} / \mathrm{h})$ between 1.82 and 26.55 with an average value of 11.91. Andesite and Marble dose rates $D(\mathrm{nGy} / \mathrm{h})$ were 24.25 and 1.97. The annual effective dose rate $D_{\text {eff }}(\mathrm{mSv} / \mathrm{y})$ ranged from 0.009 to 0.034 for Basalt and from 0.11 to 0.20 for Granite and from 0.002 to 0.033 Gold and for Andesite and for Marble were 0.029756, 0.002418 respectively. The average values were $0.022,0.17$, and 0.015 for Basalt, Granite and Gold. The External hazard index $H_{e x}$ and the internal hazard index $H_{\text {in }}$ average values were 0.099 and 0.118 for Basalt and 0.77 and 0.99 for Granite and 0.065 and 0.077 for Gold and 0.128 and 0.143 for Andesite and 0.012 and 0.016 for Marble. The average values of $H_{e x}$ and of all samples studied in this work were less than unity which means it is safe for human.

The calculated radium equivalent activities of the samples are shown in Figure 3. Maximum $R a_{e q}$ activity was calculated for sample (Granite-9) with $348.98 \mathrm{~Bq} / \mathrm{kg}$. The minimum $R a_{e q}$ activity value was calculated for sample (Gold-12) with $3.96 \mathrm{~Bq} / \mathrm{kg}$. 
Table 6. The Radium equivalent, absorbed dose rate, Annual effective dose rate, Hazard indices of all sites.

\begin{tabular}{|c|c|c|c|c|c|}
\hline Sample name & $R a_{e q}(\mathrm{~Bq} / \mathbf{k g})$ & $D(\mathrm{nGy} / \mathrm{h})$ & $D_{e f f}(\mathrm{mSv} / \mathrm{y})$ & $\boldsymbol{H}_{e x}$ & $\boldsymbol{H}_{\text {in }}$ \\
\hline Basalt-1 & 51.70325 & 25.17459 & 0.030897 & 0.139622 & 0.162649 \\
\hline Basalt-2 & 42.05116 & 19.98302 & 0.024525 & 0.113566 & 0.141431 \\
\hline Basalt-3 & 14.96213 & 07.25043 & 0.008898 & 0.040406 & 0.048622 \\
\hline Basalt-4 & 57.42034 & 27.73566 & 0.03404 & 0.155064 & 0.183577 \\
\hline Basalt-5 & 27.48013 & 13.11005 & 0.01609 & 0.074209 & 0.086885 \\
\hline Basalt-6 & 25.99559 & 12.44317 & 0.015272 & 0.070203 & 0.085203 \\
\hline Basalt-7 & 36.39555 & 17.53307 & 0.021518 & 0.098287 & 0.11772 \\
\hline Range & $14.96-57.42$ & 07.25 - 27.74 & $0.0089-0.034$ & $0.040-0.155$ & $0.049-0.184$ \\
\hline Average & 36.57259 & 17.60428 & 0.021606 & 0.098765 & 0.118012 \\
\hline Granite-8 & 328.3414 & 153.862 & 0.188835 & 0.886827 & 1.191611 \\
\hline Granite-9 & 348.9751 & 165.0165 & 0.202525 & 0.942493 & 1.202142 \\
\hline Granite-10 & 173.7983 & 85.21661 & 0.104586 & 0.469333 & 0.546143 \\
\hline Granite-11 & 288.1419 & 136.5968 & 0.167645 & 0.778217 & 1.011054 \\
\hline Range & $173.80-348.98$ & $85.22-165.02$ & $0.11-0.20$ & $0.47-0.94$ & $0.55-1.20$ \\
\hline Average & 284.8142 & 135.173 & 0.165898 & 0.769217 & 0.987738 \\
\hline Gold-12 & 3.95592 & 1.81844 & 0.002232 & 0.010683 & 0.012791 \\
\hline Gold-13 & 14.60653 & 7.36705 & 0.009042 & 0.039442 & 0.043712 \\
\hline Gold-14 & 52.81863 & 26.55153 & 0.032587 & 0.142642 & 0.174642 \\
\hline Range & $3.96-52.82$ & $1.82-26.55$ & $0.002-0.033$ & $0.011-0.143$ & $0.013-0.175$ \\
\hline Average & 23.79369 & 11.91234 & 0.01462 & 0.064256 & 0.077049 \\
\hline Andesite-15 & 47.39251 & 24.24469 & 0.029756 & 0.127975 & 0.143435 \\
\hline Marble-16 & 4.31495 & 1.97039 & 0.002418 & 0.011655 & 0.015871 \\
\hline
\end{tabular}

Comparison of the activity concentrations of ${ }^{226} \mathrm{Ra},{ }^{232} \mathrm{Th},{ }^{40} \mathrm{~K}$ and $R a_{e q}(\mathrm{~Bq} / \mathrm{kg})$ in the present work and other countries are shown in Table 7. It was observed that activity concentrations of Basalt in Yemen showed the greatest concentrations, while Basalt in Iraq, Austria and Saudi Arabia (present work) contained only less of radionuclides. Granite in Iran, Greece and present work showed high concentrations of radionuclides, while Granite in Saudi Arabia (Ryadh) and Egypt contained less of radionuclides. For Marble, the maximum activity value $56.78(\mathrm{~Bq} / \mathrm{kg})$ of ${ }^{226} \mathrm{Ra}$ was in Egypt and the maximum activity values in $(\mathrm{Bq} / \mathrm{kg}) 18.00$ and 310.00 of ${ }^{232} \mathrm{Th}$ and ${ }^{40} \mathrm{~K}$ were found in Algeria. Marble in Saudi Arabia contained only traces of radionuclides.

\section{Conclusion}

Sixteen samples of natural raw materials that are normally used in the building industry in Saudi Arabia were analyzed by the XRD analysis. The results indicate that the main constituents (major) of these samples are Albite, Anorthite and Augite) in Basalt, Albite, Microcline and Quartz in Granite, Quartz in Gold, Albite, Clinochlore and Quartz in Andesite, and Dolomite in Marble. The atomic absorption analysis results show that $\mathrm{Al} \%$ in Basalt samples has almost equal concentrations in ppm, also in Granite samples and Gold samples. For Bi and $\mathrm{U}$, the concentrations in ppm are LDL except Bi in Gold samples 12 and 13. Samples of Granite 8 and Gold 12 have the higher concentrations of $\mathrm{Pb}$. The higher concentrations of Th are observed in samples of Granite 8,9 and 11; K contains relatively high concentrations in all samples. Atomic absorption analysis results are in a good agreement with those obtained by gamma spectrometry measurements for thorium and for potassium. So, the atomic absorption analysis method is important for geochemical analysis to determine the concentrations of elements appearing in the examined minerals. The activity concentrations of ${ }^{226} \mathrm{Ra},{ }^{232} \mathrm{Th}$ and ${ }^{40} \mathrm{~K}$ have been 


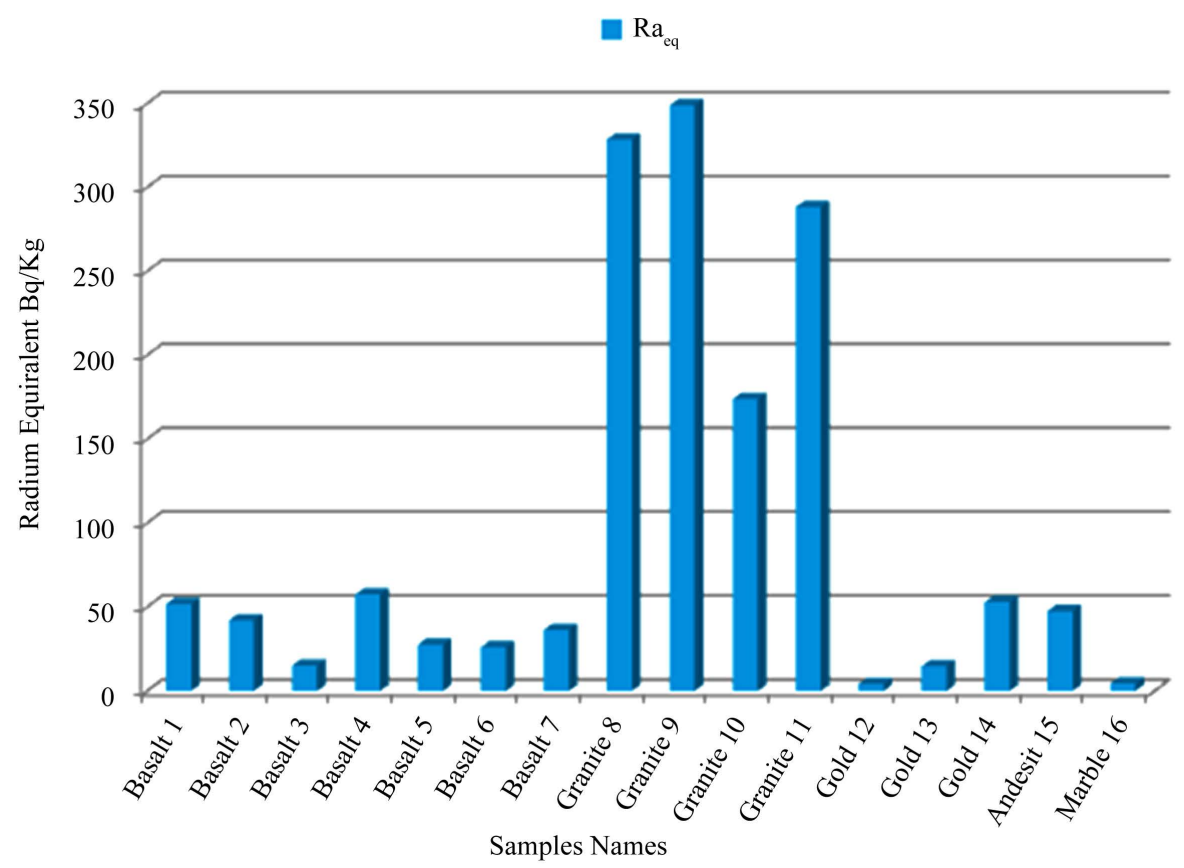

Figure 3. Radium equivalent activity value of each sample.

Table 7. Comparison of activity concentrations of ${ }^{226} \mathrm{Ra},{ }^{232} \mathrm{Th},{ }^{40} \mathrm{~K}(\mathrm{~Bq} / \mathrm{kg})$ and $R a_{e q}$ in Saudi Arabia samples and other countries.

\begin{tabular}{|c|c|c|c|c|c|c|c|}
\hline \multirow{2}{*}{ Countries } & \multirow{2}{*}{ Area } & \multirow{2}{*}{ Samples } & \multicolumn{3}{|c|}{ Activity concentrations $(\mathrm{Bq} / \mathbf{k g})$} & \multirow[b]{2}{*}{$R a_{e q}(\mathrm{~Bq} / \mathbf{k g})$} & \multirow{2}{*}{ References } \\
\hline & & & ${ }^{226} \mathrm{Ra}$ & ${ }^{232} \mathrm{Th}$ & ${ }^{40} \mathrm{~K}$ & & \\
\hline Yemen & Aden & Basalt & 57.086 & 80.26 & 846.21 & 236.04 & Harb et al., 2014* \\
\hline Iraq & Sidakan & Basalt & 5.65 & 21.4 & 203.34 & 44.8 & Ahmed and Hussein, 2011 [19] \\
\hline Austria & Austria & Basalt & 23.3 & 17.7 & 307.00 & ---- & Sorantin and Steger, 1984 [20] \\
\hline Sa. Arabia & Harrat & Basalt & 07.90 & 09.14 & 234.81 & 36.57 & Present work \\
\hline Iran & Tehran & Granite & 72.24 & 75.71 & 1126.28 & 272.32 & Asgharizadeh et al., 2012 [21] \\
\hline Sa. Arabia & Ryadh & Granite & 9.94 & 0.25 & 359.74 & 50.05 & Al-Saleh and Al-Berzan, 2007 [22] \\
\hline Egypt & Aswan & Granite & 14.94 & 15.50 & 370.23 & 35.73 & El-Taher et al., 2007 [23] \\
\hline Greece & Greece & Granite & 64.56 & 85.53 & 1104.19 & ---- & Papastefanou et al., 2005 [24] \\
\hline Sa. Arabia & Alquryyah & Granite & 80.85 & 68.50 & 1376.69 & 284.81 & Present work \\
\hline Egypt & Sinai, Suez & Marble & 56.78 & 5.95 & 142.8 & 76.29 & Fares et al., 2011** \\
\hline Sa. Arabia & Sa. Arabia & Marble & 12.00 & 1.70 & 23.10 & 16.211 & Al-Saleh and Al-Berzan, 2007*** \\
\hline Algeria & Product & Marble & 23.00 & 18.00 & 310.00 & 73.00 & Amrani and Tahtat, $2001 * * * *$ \\
\hline Sa. Arabia & Madrekah & Marble & 01.56 & 01.38 & 10.15 & 4.32 & Present work \\
\hline
\end{tabular}

$*[4] ; * *[3] ; * *[22] ; * * * *[8]$.

found using gamma-ray spectrometry. The concentrations ${ }^{226} \mathrm{Ra}$ and ${ }^{40} \mathrm{~K}$ in Granite samples are among the highest measured values, while in Marble, ${ }^{226} \mathrm{Ra},{ }^{232} \mathrm{Th}$ and ${ }^{40} \mathrm{~K}$ are the lowest measured values compared with those of other countries. The average values of external and internal hazard indices of all samples are less than unity. The activity concentrations of these samples produce an average radium equivalent activity between 3.96 and 284.81 which is less than that recommended by UNSCEAR $(370 \mathrm{~Bq} / \mathrm{kg})$. The highest average value of the absorbed dose $135.17(\mathrm{nGy} / \mathrm{h})$ is in Granite which is higher than the estimate of average global terrestrial. The ef- 
fective dose rate $(\mathrm{mSv} / \mathrm{y})$ values are less than unity for all samples which are acceptable global values. All results are within the range as given in UNSCEAR.

\section{Acknowledgements}

The author is indebted to the Saudi Geological Survey (SGS) for supplying the geological samples, also for their technical help.

\section{References}

[1] Mineral Data (2012) Webmineral.com.

[2] Mineral Resources and Mining. http://www.saudinf.com/main/e7.htm

[3] Fares, S., Yassene Ali, A.M., Ashour, A., Abu-Assy, M.K. and Abd El-Rahman, M. (2011) Natural Science, 3, 895905. http://dx.doi.org/10.4236/ns.2011.310115

[4] Harb, S., El-Kamel, A.H., Zahran, A.M., Abbady, A. and Ahmed, F.A. (2014) International Journal of Mathematics and Physical Sciences Research (IJMPSR), 1, 7-17.

[5] Asgharizadeh, F., Abbasi, A., Hochaghani, O. and Gooya, E.S. (2012) Radiation Protection Dosimetry, 149, $321-326$. http://dx.doi.org/10.1093/rpd/ncr233

[6] Ministry of Petroleum and Mineral Resources, Kingdom of Saudi Arabia (2009) http://www2.mopm.gov.sa/minerals.html

[7] Saudi Geological Survey (SGS) (2012) http://www.sgs.org.sa

[8] Amrani, D. and Tahtat, M. (2001) Applied Radiation and Isotopes, 54, 687-689. http://dx.doi.org/10.1016/S0969-8043(00)00304-3

[9] Tufail, M. and NasimAkhtar, M. (2006) Radiation Measurements, 41, 443-451. http://dx.doi.org/10.1016/j.radmeas.2005.10.007

[10] UNSCEAR (2000) United Nations Scientific Committee on the Effects of Atomic Radiation. Sources and Effects of Ionizing Radiations. United Nations, New York.

[11] Veiga, R., Sanches, N., Anjos, R.M., Macario, K., Bastos, J., Iguatemy, M., Aguiar, J.G., Santos, A.M.A., Mosquera, B., Carvalho, C., Baptista, F.M. and Umisedo, N.K. (2006) Radiation Measurements, 41, 189-196. http://dx.doi.org/10.1016/j.radmeas.2005.05.001

[12] El Aassy, I.E., El Galy, M.M., Nada, A.A., El Feky, M.G., El Maksoud, T.M.A., Talaat, S.M. and Ibrahim, E.M. (2011) Journal of Radioanalytical and Nuclear Chemistry, 289, 173-184. http://dx.doi.org/10.1007/s10967-011-1059-1

[13] Nada, A. (2003) Applied Radiation and Isotopes, 58, 275-280. http://dx.doi.org/10.1016/S0969-8043(02)00258-0

[14] Harris, W. and White, G.N. (2008) X-Ray Diffraction Techniques for Soil Mineral Identification. In: Ulery, A.L. and Drees, L.R., Eds., Methods of Soil Analysis Part 5-Mineralogical Methods, American Society of Agronomy, S. Segoe, Madison, 677.

[15] Leet, L., Judson, S. and Kauffman, M. (1982) Physical Geology. 6th Edition, Englewood Cliffs.

[16] Mineral Data, Webmineral.com, 2012.

[17] International Atomic Energy Agency (1989) Construction and Use of Calibration Facilities for Radiometric Field Equipment. Technical Reports Series No. 309, IAEA, Vienna.

[18] UNSCEAR (1993) Sources and Effects of Ionizing Radiation. United Nations Scientific Committee on the Effects of Atomic Radiation. Report to the General Assembly, with Scientific Annexes, United Nations, New York.

[19] Ahmed, A.A. and Hussein, M.I. (2011) World Academy of Science, Engineering and Technology, 5, 2-20.

[20] Sorantin, H. and Steger, F. (1984) Radiation Protection Dosimetry, 7, $59-61$.

[21] Asgharizadeh, F., Abbasi, A., Hochaghani, O. and Gooya, E.S. (2012) Radiation Protection Dosimetry, 149, $321-326$. http://dx.doi.org/10.1093/rpd/ncr233

[22] Al-Saleh, F.S. and Al-Berzan, B. (2007) Journal of Nuclear and Radiation Physics, 2, 25-36.

[23] El-Taher, A., Uosif, M.A.M. and Orabi, A.A. (2007) Radiation Protection Dosimetry, 124, 148-154. http://dx.doi.org/10.1093/rpd/ncm211

[24] Papastefanou, C., Stoulos, S. and Manolopoulou, M. (2005) Journal of Radioanalytical and Nuclear Chemistry, 266, 367-372. http://dx.doi.org/10.1007/s10967-005-0918-z 